\title{
Mucosite oral relacionada à quimioterapia em pacientes com câncer de mama: uma breve revisão
}

\author{
Chemotherapy-related oral mucositis in breast cancer patients: a brief review \\ Mucositis oral relacionada con la quimioterapia en pacientes con cáncer de mama: una breve \\ revisión
}

Pabliane Matias Lordelo Marinho ORCID: https://orcid.org/0000-0001-6190-0844 Universidade de São Paulo, Brasil E-mail: marinho.pabliane@gmail.com Ricardo Barbosa Lima ORCID: https://orcid.org/0000-0001-5274-4800 Universidade de São Paulo, Brasil E-mail: ricardobarbosalima@usp.br

José Cleyton de Oliveira Santos ORCID: https://orcid.org/0000-0001-5616-7625 Universidade Federal de Sergipe, Brasil E-mail: cleyton-121@ @otmail.com

Dayane Ketlyn da Cunha Santos ORCID: https://orcid.org/0000-0002-9312-4891 Universidade Federal de Sergipe, Brasil E-mail: daayketlyn27@gmail.com

Glebson Santos Sobral

ORCID: https://orcid.org/0000-0002-3835-7916 Universidade Federal de Sergipe, Brasil E-mail: sobral.glebson@hotmail.com

Andressa Cabral Vassilievitch

ORCID: https://orcid.org/0000-0002-2421-4607 Universidade Federal de Sergipe, Brasil E-mail: andressavassi98@gmail.com

Bruno Ferreira Amorim

ORCID: https://orcid.org/0000-0002-2581-2983 Universidade Federal de Sergipe, Brasil E-mail: brunofamorim@hotmail.com.br Jéssica dos Santos Costa

ORCID: https://orcid.org/0000-0003-2404-6604 Instituto de Medicina Integral Professor Fernando Figueira, Brasil E-mail: jessicas_costa@outlook.com

Tiago Vasconcelos Fonseca

ORCID: https://orcid.org/0000-0002-7632-9860 Universidade Federal de Sergipe, Brasil E-mail: tiagovasconfonseca@gmail.com

Glebson Moura Silva

ORCID: https://orcid.org/0000-0002-4977-2787 Universidade Federal de Sergipe, Brasil E-mail: glebsonmoura@yahoo.com.br

Simone Yuriko Kameo

ORCID: https://orcid.org/0000-0002-0035-2415 Universidade Federal de Sergipe, Brasil

E-mail: simonekameo@ hotmail.com

Namie Okino Sawada

ORCID: https://orcid.org/0000-0002-1874-3481 Universidade Federal de Alfenas, Brasil

E-mail: namie.sawada@unifal-mg.edu.br

\section{Resumo}

O câncer de mama pode impactar diversas esferas de saúde dos pacientes, uma vez que a vivência frente ao tratamento é frequentemente cercada de medos e anseios. É possível observar que reações adversas da quimioterapia são comuns e mulheres com câncer de mama podem apresentar alta incidência de mucosite oral (MO). Sendo assim, o objetivo desta 
revisão foi discutir a relação entre o tratamento quimioterápico do câncer de mama e a ocorrência da mucosite oral. A MO é caracterizada pelo surgimento de eritemas ou úlceras na cavidade oral, sendo que a sua classificação varia conforme a escala utilizada. A fisiopatologia está associada aos fatores de risco, visto que o tratamento administrado e o estilo de vida do paciente podem impactar diretamente na ocorrência e progressão da MO. Embora a maioria dos casos apresenta-se como leve ou moderada, o impacto da MO não pode ser subestimado, mesmo em casos de baixa sintomatologia ou impacto funcional, considerando a dor oral e a dificuldade na ingestão de alimentos. Assim, é importante que os profissionais estejam atentos à classificação, fatores de risco e possíveis impactos na saúde, incluindo o planejamento das intervenções preventivas e curativas no manejo desta reação adversa da quimioterapia. Profissionais de saúde, incluindo dentistas, enfermeiros e médicos devem estar preparados para lidar com casos de MO, promovendo ações integradas para reduzir o impacto dessa condição na saúde de mulheres com câncer de mama.

Palavras-chave: Estomatite; Neoplasias da mama; Quimioterapia; Antineoplásicos.

\begin{abstract}
Breast cancer can impact different health spheres of patients, since the experience with the treatment is often surrounded by fears and anxieties. It is possible to observe that adverse effects of chemotherapy are common and women with breast cancer may have a high incidence of oral mucositis (OM). Thus, the objective of this review was to discuss the relationship between chemotherapy treatment of breast cancer and the occurrence of oral mucositis. OM is characterized by the appearance of erythema or ulcers in the oral cavity, and its classification varies according to the scale used. The pathophysiology is associated with risk factors, since the treatment administered and the patient's lifestyle can directly impact the occurrence and progression of OM. Although most cases are mild or moderate, the impact of OM can not be underestimated, even in cases of low symptoms or functional impact, considering oral pain and difficulty in food intake. Thus, it is important that professionals pay attention to the classification, risk factors and possible impacts on health, including the planning of preventive and curative actions in the management of this adverse effect of chemotherapy. Health professionals, including dentists, nurses and doctors must be prepared to deal with cases of OM, promoting integrated actions to reduce the impact of this condition on the health of women with breast cancer.
\end{abstract}

Keywords: Stomatitis; Breast neoplasms; Chemotherapy; Antineoplastic agents.

\title{
Resumen
}

El cáncer de mama puede impactar en distintos ámbitos de la salud de las pacientes, ya que la experiencia con el tratamiento suele estar rodeada de miedos y ansiedades. Es posible observar que los efectos adversos de la quimioterapia son comunes y las mujeres con cáncer de mama pueden tener una alta incidencia de mucositis oral (MO). Por lo tanto, el objetivo de esta revisión fue discutir la relación entre el tratamiento con quimioterapia del cáncer de mama y la aparición de mucositis oral. La MO se caracteriza por la aparición de eritema o úlceras en la cavidad bucal, y su clasificación varía según la escala utilizada. La fisiopatología está asociada a factores de riesgo, ya que el tratamiento administrado y el estilo de vida del paciente pueden impactar directamente en la aparición y progresión de la OM. Aunque la mayoría de los casos son leves o moderados, el impacto de la MO no puede subestimarse, incluso en casos de síntomas bajos o impacto funcional, considerando el dolor bucal y la dificultad para ingerir alimentos. Por ello, es importante que los profesionales estén atentos a la clasificación, los factores de riesgo y los posibles impactos en la salud, incluida la planificación de intervenciones preventivas y curativas en el manejo de este efecto adverso de la quimioterapia. Los profesionales de la salud, incluidos dentistas, enfermeras y médicos, deben estar preparados para atender los casos de OM, promoviendo acciones integradas para reducir el impacto de esta condición en la salud de las mujeres con cáncer de mama.

Palabras clave: Estomatitis, Neoplasias de la mama, Quimioterapia, Antineoplásicos.

\section{Introdução}

Dentre diversas doenças que acometem a saúde dos seres humanos, o câncer possui altas taxas de mortalidade no mundo.

Considerando os tipos de câncer, as neoplasias mamárias estão entre as mais prevalentes, representando um fator significativo nas taxas de mortalidade de mulheres em diversos países. Em 2018, verificou-se que o câncer de mama era o segundo mais incidente no mundo, com 2,1 milhões de casos novos, sendo o subtipo mais incidente nas mulheres (24,2\%). No Brasil, esperase que 66.280 novos casos incidam entre 2020 e 2022 (Azamjah et al., 2019; Brasil, 2019).

Para tratar o câncer de mama, diversas estratégias têm sido investigadas. Além de curar a doença, espera-se que a terapia contribua para a preservação da qualidade de vida e aumento da expectativa de vida após o diagnóstico. Essas terapias envolvem abordagens cirúrgicas, radioterapia, terapia hormonal direcionada e quimioterapia, visando a remoção e/ou destruição de células e tecidos tumorais, controlando a doença. Entretanto, todas as terapias podem desencadear reações adversas que, se não manejadas adequadamente, podem comprometer a cura do paciente (Akram et al., 2017; Bonilla et al. 2017). 
A mucosite oral é uma reação adversa na mucosa bucal desencadeada pelo efeito citotóxico dos agentes quimioterápicos nessa cavidade, podendo acometer mais de $85 \%$ dos pacientes em algumas situações clínicas, sendo comum em mulheres com câncer de mama em tratamento. Os dados epidemiológicos dessa condição podem estar subestimados em diversos estudos, embora seus impactos no tratamento oncológico e na saúde dos pacientes acometidos estejam difundidos na literatura, à medida que as características que levam ao surgimento, gravidade e cura dessa condição são amplas (Lalla et al., 2008; Barbosa-Lima et al., 2020; Pulito et al., 2020). Diante deste contexto, o objetivo desta revisão narrativa é discutir a relação entre o tratamento quimioterápico do câncer de mama e a ocorrência de mucosite oral.

\section{Metodologia}

Trata-se de uma revisão narrativa da literatura. Os artigos que compuseram o escopo de referências foram selecionados por conveniência dos autores, sem um método de busca específico (Rother, 2007; Thorne, 2018). Todos os artigos incluídos foram aplicados em direção ao objetivo do estudo, visando construir uma revisão da literatura focada na problemática abordada: a relação entre a mucosite oral provocada pelos agentes antineoplásicos e as pacientes em tratamento quimioterápico para o câncer de mama, abordando diversos aspectos desta interface.

\section{Revisão de Literatura}

\subsection{A mucosite oral}

A mucosite oral é caracterizada pelo surgimento de alterações eritematosas ou ulcerativas nos tecidos moles da cavidade oral. É uma condição frequentemente associada ao desconforto e a dor, bem como influencia diretamente na capacidade dos pacientes de ingerir alimentos sólidos, sendo um ponto crítico no impacto da mucosite oral no tratamento oncológico, pois o comportamento e a sintomatologia das lesões determinam as intervenções para a manutenção da saúde (Lalla et al., 2008; Curra et al., 2018).

Os danos na cavidade oral frequentemente acometem as regiões da mucosa bucal, assoalho da boca, palato mole e bordas da língua. Quando outras áreas são afetadas, requerem investigações adicionais para determinar a etiologia. Além disso, é importante evidenciar que as úlceras relacionadas à mucosite oral apresentam bordas com limites mal definidos e sem anel periférico, o que diferencia as mesmas de outras condições bucais. Além disso, a mucosite oral induzida por quimioterapia tende a apresentar um comportamento mais limitado, com início nas primeiras semanas e cura em duas semanas subsequentes ao surgimento. Por outro lado, a mucosite oral induzida por radiação possui um curso mais extenso e dependente do fim da radioterapia (Lalla et al., 2008; Kusiak et al., 2020). Independente da origem, a mucosite oral associada ao tratamento oncológico pode acometer até $20-40 \%$ dos pacientes que recebem quimioterapia convencional, $80 \%$ dos pacientes que recebem altas doses e até $100 \%$ dos pacientes irradiados para câncer na região da cabeça e pescoço (Daugèlaitẻ et al., 2019).

Dentro desse âmbito epidemiológico, nota-se que o percentual de pacientes afetados aumenta para cerca de $60-85 \%$ em indivíduos submetidos a transplantes de células tronco-hematopoiéticas. Ao verificar essa manifestação em pacientes que realizam a quimioterapia adjuvante, a mucosite em relação aos pacientes que não realizaram esse procedimento, foi 50\% maior (Morais et al., 2015; Sonis, 2009; Pereira et al., 2019).

Ao analisar o panorama geral das manifestações oncológicas frente ao gênero, questões como péssimos hábitos de higiene e autocuidado, além de uma menor busca por serviços de saúde bucal, podem influenciar na manifestação da mucosite oral em pacientes do sexo masculino (Pereira et al., 2019). Contudo, frente à neoplasia da mama, fica explícito que as pacientes do sexo feminino possuem mais chances de manifestar essa reação adversa devido à alta incidência desse tipo de câncer nessa população (Araújo et al., 2015). Contudo, torna-se importante evidenciar que, frequentemente, apenas os estágios avançados de 
mucosite oral são registrados, principalmente pela necessidade de tratamento clínico e assistência (Pulito et al., 2020).

Os fatores de risco para o desenvolvimento da mucosite oral ainda não estão completamente elucidados, entretanto, as características dos agentes antineoplásicos (mecanismo de ação, dose e quantidade de ciclos) estão fortemente associados com a ocorrência e gravidade das lesões. Além disso, mulheres podem ter piores experiências com a mucosite oral, assim como pessoas com idade avançada e com saúde e higiene bucal comprometidas (Curra et al., 2018; Saedi et al., 2019; Kusiak et al., 2020).

Esses fatores são definidos como condições e/ou cenários que aumentam a probabilidade de a pessoa desenvolver a mucosite oral, desse modo, salienta-se que associados à fisiopatologia, esses norteiam os profissionais nas condutas e cuidados prestados ao paciente, com a finalidade de reduzir os impactos ocasionados por reações adversas associadas ao tratamento antineoplásico (Raber-Durlacher et al., 2010; Scully et al., 2006). O quadro 1 evidencia os fatores encontrados na literatura.

Quadro 1 - Fatores de risco para mucosite oral.

\begin{tabular}{|c|c|c|}
\hline FATOR DE RISCO & CRITÉRIO DE AVALIAÇÃO & REFERÊNCIA \\
\hline \multicolumn{3}{|c|}{ Relacionados ao tratamento } \\
\hline Tipo de tratamento & $\begin{array}{c}\text { Esquemas com 5-fluorouracil, Trastuzumabe ou } \\
\text { Paclitaxel* e associação com a radioterapia } \\
\text { apresentam maior risco }\end{array}$ & \multirow{4}{*}{$\begin{array}{c}\text { PULITO et al., } 2020 . \\
\text { CURRA et al., } 2018 . \\
\text { SONIS \& CLARK, } 1991 . \\
\text { SEILER et al., } 2014 .\end{array}$} \\
\hline Dose & $\begin{array}{l}\text { Doses maiores que as convencionais têm maior } \\
\text { risco }\end{array}$ & \\
\hline Via de administração & Endovenosa & \\
\hline Tipos de tumores & $\begin{array}{l}\text { Tumores sólidos apresentam maior risco, } \\
\text { principalmente na área próxima à cavidade oral }\end{array}$ & \\
\hline \multicolumn{3}{|c|}{ Relacionados ao paciente } \\
\hline Idade & Extremos de idades (crianças e idosos) & \multirow{4}{*}{$\begin{array}{c}\text { CHAVELI-LÓPEZ; BAGÁN- } \\
\text { SEBASTIÁN, } 2016 . \\
\text { BARASH \& PETERSON, } 2003 \\
\text { ARAÚJO et al, } 2015 \\
\text { PEREIRA et al., } 2019 \\
\text { RABER-DURLACHER; ELAD; } \\
\text { BARASCH, } 2010\end{array}$} \\
\hline \multirow[t]{2}{*}{ Gênero } & $\begin{array}{c}\text { Feminino* } \\
\text { Pacientes do sexo feminino possuem maior risco de } \\
\text { desenvolver mucosite quando tratados com 5- } \\
\text { fluoruracil. } \\
\text { Maior incidência de câncer de mama nessa } \\
\text { população }\end{array}$ & \\
\hline & $\begin{array}{l}\text { Masculino** } \\
\text { Maior negligência com saúde bucal e autocuidado }\end{array}$ & \\
\hline $\begin{array}{l}\text { Índice de massa corporal } \\
(I M C)\end{array}$ & Menor que 20 & \\
\hline Prótese dentária & Uso de prótese dentária & MCCARTHY et al., 1998 \\
\hline Educação & Menor nível educacional aumenta o risco & \\
\hline
\end{tabular}




\begin{tabular}{|c|c|c|}
\hline Pobre higiene oral & Escovação menor que duas vezes ao dia & SONIS et al., 1978 \\
\hline Comorbidades & $\begin{array}{c}\text { Diabetes Mellitus, Doença de Addison e disfunção } \\
\text { renal }\end{array}$ & $\begin{array}{c}\text { RABER-DURLACHER; ELAD; } \\
\text { BARASCH, } 2010\end{array}$ \\
\hline Leucócitos & Abaixo de $3.000 / \mathrm{uL}$ & SURESH et al., 2010 \\
\hline Álcool & Uso de álcool atual ou anterior ao tratamento & PATUSSI et al., 2014. \\
\hline Tabagismo & $\begin{array}{l}\text { Uso de tabaco atual ou anterior ao tratamento. } \\
\text { Pacientes tabagistas apresentaram graus mais } \\
\text { elevados de mucosite oral radioinduzida diante o } \\
\text { momento do surgimento }\end{array}$ & $\begin{array}{c}\text { DODD et al., } 1999 \\
\text { HOLMES et al., } 2014\end{array}$ \\
\hline Fatores genéticos & $\begin{array}{l}\text { Polimorfismos genéticos relacionados com a } \\
\text { expressão de mediadores inflamatórios, a exemplo } \\
\text { do TNF-alfa. } \\
\text { Utilização de drogas que afetam a síntese de DNA, } \\
\text { como os antimetabólitos. } \\
\text { Pacientes com deficiência da dihidropirimidina } \\
\text { desidrogenase, enzima fundamental para o } \\
\text { catabolismo de drogas. }\end{array}$ & $\begin{array}{c}\text { SONIS, } 2010 \\
\text { CHAVELI-LÓPEZ; BAGÁN- } \\
\text { SEBASTIÁN, } 2016 \\
\text { SLOAN et al., } 2002\end{array}$ \\
\hline $\begin{array}{l}\text { Mucosite no primeiro ciclo } \\
\text { de quimioterapia }\end{array}$ & $\begin{array}{l}\text { Lesão em qualquer grau após a primeira sessão de } \\
\text { quimioterapia. }\end{array}$ & SONIS, 2009 \\
\hline Má saúde bucal & Doença periodontal & AL-ANSARI et al., 2015 \\
\hline Trauma da mucosa & $\begin{array}{l}\text { Trauma pode contribuir para o desenvolvimento da } \\
\text { mucosite oral. }\end{array}$ & $\begin{array}{c}\text { RABER-DURLACHER; ELAD; } \\
\text { BARASCH, } 2010\end{array}$ \\
\hline
\end{tabular}

*Analisando o panorama do câncer de mama.

**Analisando o panorama geral dos cânceres em relação ao autocuidado masculino. Fonte: Autores.

As variáveis do tratamento podem afetar a incidência e gravidade da mucosite, dessa forma, deve-se estar atento a fatores diretamente relacionados à terapia, como tratamento, medicamentos e doses. Além de investigar sobre os riscos intrínsecos ao paciente, como condições médicas pré-existentes, alterações da dinâmica bucal e estado geral, idade, saúde bucal (higiene diante o tratamento), estado nutricional do paciente e o seu funcionamento hepático e renal são fundamentais, devendo o profissional estar atento a toda essa esfera (Al-Ansari et al., 2015; Chavelli-López \& Bagán-Sebastián, 2016).

Os impactos da mucosite oral aos pacientes com câncer são significativos e podem provocar alterações no tratamento, como redução das doses, uso de terapias de segunda linha, infecções secundárias e, em casos graves, interrupção do mesmo. Além disso, pode haver aumento nas internações e custos adicionais com o cuidado destes pacientes. Esse conjunto de consequências podem impactar diretamente na sobrevida dos pacientes com câncer (Lalla et al., 2008; Kusiak et al., 2020). 


\subsection{Fisiopatologia da mucosite oral}

A fisiopatologia da mucosite oral é considerada um evento complexo. Com o dano provocado ao DNA pelos agentes quimioterápicos, considerando que estes não conseguem distinguir as células malignas altamente proliferativas das células da camada basal do epitélio, o turnover celular é comprometido. Após o dano, uma cascata de sinalizações celulares desencadeia uma resposta inflamatória que resulta no surgimento das lesões na cavidade oral, dando início ao curso da doença (Shankar et al., 2017; Pulito et al., 2020).

A ativação do fator nuclear kappa B (NF-kB), um fator de transcrição, é um dos principais mecanismos para a sinalização celular e amplificação, geralmente desencadeada pela liberação de espécies reativas de oxigênio pelas células danificadas por agentes quimioterápicos citotóxicos. Esta ativação ocorre junto à liberação de moléculas DAMPs (padrões moleculares associados ao dano), cuja atuação conjunta levam à transcrição de citocinas mediadas por NF-kB, como fator de necrose tumoral alfa (TNF-a), interleucina 6 (IL-6) e interleucina 1 beta (IL-1B), caracterizando a reação pró-inflamatória (Lalla et al., 2008; Shankar et al., 2017; Pulito et al., 2020).

Posteriormente, TNF-a realiza um feedback positivo para NF-kB, sustentando a ativação deste fator durante a progressão da mucosite oral, ativando também a sinalização e liberação de proteínas quinase ativadas por mitógenos (MAPK), que leva à ativação de JNK (c-Jun N-terminal quinase), levando à morte celular. Além disso, pode haver aumento da expressão de metaloproteinases de matriz 1 e 3 (MMP-1 e MMP-3) pelos fibroblastos danificados (Shankar et al., 2017; Pulito et al., 2020).

Todos estes eventos ocorrem em cinco fases distintas: 1. Iniciação (danos ao DNA celular e liberação de espécies reativas de oxigênio), 2. Reação inflamatória (regulação positiva de citocinas pró-inflamatórias), 3. Sinalização e amplificação (citocinas pró-inflamatórias já liberadas ativam outras vias que aumentam a liberação de outras citocinas e ativam vias que levam ao dano celular, 4. Ulceração e inflamação (as lesões interagem com o meio bucal e seus microrganismos, aumentando a quantidade de células inflamatórias e regulando positivamente a expressão de citocinas pró-inflamatórias) e 5. Cicatrização e cura (restabelecimento da integridade epitelial) (Lalla et al., 2008; Shankar et al., 2017).

\subsection{Avaliação da mucosite oral}

Dois instrumentos estão disponíveis e difundidos para avaliação da manifestação da mucosite oral enquanto reação adversa provocada pelo tratamento oncológico. A mais utilizada é a World Health Organization - Oral Mucositis Grading Scale (WHO-OMGS), que adota critérios clínicos relacionados à lesão de mucosite oral e a capacidade de ingerir alimentos. Por outro lado, o critério da Common Terminology Criteria for Adverse Events, em sua quinta versão (CTCAE v5.0), considera aspectos relacionados ao impacto da mucosite oral: intensidade da dor, capacidade de ingerir alimentos e necessidade de intervenção (Lalla et al., 2008; Chaveli-López \& Bagán-Sebastián, 2016; Al-Ibraheemi \& Shamoun, 2016; National Cancer Institute, 2017). O Quadro 2 descreve a classificação da mucosite oral de acordo com estes dois critérios. 
Quadro 2. Classificação da mucosite oral de acordo com o CTCAE v5.0 e WHO-OMGS.

\begin{tabular}{|c|c|c|}
\hline Estágio & Critério CTCAE v5.0 & Critério WHO-OMGS \\
\hline $1 / \mathrm{I}$ & $\begin{array}{c}\text { Pacientes assintomáticos ou com sintomas leve } \\
\text { e que não necessitam de intervenção }\end{array}$ & Dor oral e eritema \\
\hline $2 / \mathrm{II}$ & $\begin{array}{c}\text { Pacientes com dor moderada ou úlceras que } \\
\text { não interferem na ingestão de alimentos, } \\
\text { necessitando apenas de modificações na dieta }\end{array}$ & Eritemas ou úlceras orais, mas toleram \\
alimentos sólidos
\end{tabular}

Fonte: dados extraídos do Common Terminology Criteria for Adverse Events, em sua quinta versão (CTCAE v5.0) e do artigo de Al Ibraheemi \& Shamoun (2016), que descrevem os critérios WHO-OMGS.

\subsection{Quimioterapia para o câncer de mama e mucosite oral}

Diversos agentes quimioterápicos utilizados no câncer de mama apresentam risco para o desenvolvimento de mucosite oral. Os principais agentes neste tipo de tumor sólido são doxorrubicina, ciclofosfamida, taxanos (como paclitaxel ou docetaxel) e 5-fluorouracil, combinados em diferentes regimes. Investigações sobre o uso destes agentes e a ocorrência de mucosite oral já descrevem alta incidência desta condição em mulheres com câncer de mama expostas (Seiler et al., 2014; Hong et al., 2019a; Barbosa-Lima et al., 2020).

Entretanto, embora haja conhecimento deste fenômeno, ainda faltam estudos mais acurados sobre a exposição aos agentes antineoplásicos e seus protocolos (combinações, doses e quantidade de ciclos) com o desenvolvimento da mucosite oral e outras toxicidades bucais. Além disso, também são necessários estudos clínicos mais acurados para propor adequações nos protocolos quimioterápicos visando a prevenção desta condição em pacientes oncológicos (Jesus et al., 2016; Curra et al., 2018).

Previamente, uma evidência sugeriu que não haviam diferenças na incidência de estomatites relacionadas à quimioterapia para o câncer de mama em mulheres. Por outro lado, há evidência de que protocolos envolvendo doxorrubicina e ciclofosfamida (weekly AC protocol) podem estar associados à uma maior chance de desenvolver mucosite oral em estágios $3 \mathrm{e}$ 4 em relação aos demais protocolos, seguido pela combinação AT (doxorrubicina e paclitaxel ou docetaxel) (Seiler et al., 2014; Zhang et al., 2016).

Comparando a incidência de estomatites em relação aos agentes taxanos, um estudo retrospectivo em prontuários clínicos identificou que a exposição à agentes taxanos em algum momento da quimioterapia para câncer de mama esteve associada ao maior risco de manifestar mucosite oral, embora a maioria das pacientes tenham desenvolvido grau 1 ou 2. Entre os agentes taxanos, considerando paclitaxel e docetaxel, parece não haver diferenças nesse desfecho (Watanabe et al., 2017; Barbosa-Lima et al., 2020). 


\subsection{Manejo da mucosite oral}

Diversas estratégias têm sido utilizadas para o manejo da mucosite oral em pacientes que realizam quimioterapia para o tratamento do câncer, incluindo medidas de prevenção e tratamento. Na literatura é possível encontrar diretrizes recentes para a prevenção e o manejo desta condição, incluindo a atualização da diretriz elaborada pela Multinational Association of Supportive Care in Cancer e pela International Society of Oral Oncology (MASCC/ISOO), da autoria de Elad e colaboradores (2020) (Elad et al., 2020; Wilairat et al., 2020).

As intervenções para manejar a mucosite oral podem ser subdivididas em oito linhas de atuação: cuidados básicos com a cavidade oral, uso de agentes anti-inflamatórios, fotoestimulação (luz e laser), crioterapia, agentes antimicrobianos/anestésicos/analgésicos (incluindo coberturas), fatores de crescimento e citocinas, produtos naturais e outras intervenções para mucosites no trato gastrointestinal (Elad et al., 2020).

Considerando a mucosite oral associada à quimioterapia, a diretriz MASCC/ISOO recomenda a higiene oral básica com vários agentes combinados para prevenção da mesma. Entretanto, reporta ainda que não há evidências consistentes para indicar higiene oral profissional, bem como pontua que soluções salinas, com bicarbonato de sódio ou clorexidina também não apresentam evidências robustas para serem recomendadas pela diretriz (Hong et al., 2019b; Elad et al., 2020).

Por outro lado, outras metanálises de ensaios clínicos randomizados identificaram que tanto a clorexidina quanto outras substâncias sintéticas (benzidamina e sucralfato) e naturais (mel e curcumina) podem ser benéficas no manejo da mucosite oral quando usadas como soluções de higiene oral/colutórios (Yu et al., 2020; Zhang et al., 2020). Por outro lado, considerando os agentes anti-inflamatórios e produtos naturais, a diretriz MASCC/ISOO recomenda apenas benzidamina para pacientes com câncer de cabeça e pescoço que recebem quimiorradioterapia, sem recomendações mais expressivas para pacientes com tumores sólidos que receberam apenas quimioterapia (Ariyawardana et al., 2019; Elad et al., 2020).

Em relação à crioterapia, a diretriz MASCC/ISOO recomenda esta modalidade terapêutica apenas aos pacientes que receberam quimioterapia com 5-fluorouracil administrado em bolus. Entretanto, outras evidências indicam o uso desta modalidade para a prevenção da mucosite oral em pacientes oncológicos, considerando-a uma das intervenções mais eficazes e seguras neste contexto, embora ensaios clínicos de maior impacto ainda sejam necessários (Park \& Lee, 2019; Elad et al., 2020; Wilairat et al., 2020).

A higiene bucal é uma intervenção simples e de baixo custo que atua reduzindo a carga bacteriana e diminuindo a probabilidade de complicações associadas. Desse modo, a articulação do profissional e paciente é fundamental para a orientação correta quanto à realização dessa prática e seus impactos para o manejo da mucosite e promoção de bem-estar no paciente (Elad et al., 2020).

\section{Conclusão}

A ocorrência de mucosite oral é um fenômeno complexo e demanda de atenção pelas equipes de saúde em serviços de oncologia para a melhor prática baseada em evidências no manejo desta condição, bem como pelos pesquisadores, orientando a busca de conhecimentos necessários para uma melhor compreensão dos eventos que levam ao surgimento da mucosite oral e das intervenções mais efetivas para prevenir e tratar.

\section{Referências}

Akram, M., Iqbal, M., Daniyal, M., \& Khan, A. U. (2017). Awareness and current knowledge of breast cancer. Biological Research, 50(1), 1-23. 
Al Ibraheemi, A. A., \& Shamoun, S. (2016). Incidence and risk factors of oral mucositis in patients with breast cancer who receiving chemotherapy in Al-Bashir hospital. International Journal of Hematology-oncology and Stem Cell Research, 10(4), 217.

Araújo, S. N. M., Luz, M. H. B. A., Silva, G. R. F. D., Andrade, E. M. L. R., Nunes, L. C. C., \& Moura, R. O. (2015). Cancer patients with oral mucositis: challenges for nursing care. Revista Latino-americana de Enfermagem, 23(2), 267-274.

Ariyawardana, A., Cheng, K. K. F., Kandwal, A., Tilly, V., Al-Azri, A. R., Galiti, D., \& Elad, S. (2019). Systematic review of anti-inflammatory agents for the management of oral mucositis in cancer patients and clinical practice guidelines. Supportive Care in Cancer, 27(10), 3985-3995.

Azamjah, N., Soltan-Zadeh, Y., \& Zayeri, F. (2019). Global trend of breast cancer mortality rate: a 25-year study. Asian Pacific Journal of Cancer Prevention: APJCP, 20(7), 2015.

Barasch, A., \& Peterson, D. E. (2003). Risk factors for ulcerative oral mucositis in cancer patients: unanswered questions. Oral Oncology, 39(2), 91-100.

Barbosa-Lima, R., Kameo, S. Y., Amorim, B. F., Ramos, M. J. O., dos Santos Costa, J., Marinho, P. M. L., \& Silva, G. M. (2020). Occurrence of oral mucositis in women during oncological treatment of breast cancer in the Brazilian Northeast. Health Sciences Journal, 10(4), $144-150$.

Brasil. (2019). Estimativa 2020: Incidência de Câncer no Brasil. Instituto Nacional de Câncer - José de Alencar Gomes da Silva. Rio de Janeiro: Ministério da Saúde.

Bonilla, J. M., Tabanera, M. T., \& Mendoza, L. R. (2017). Breast cancer in the 21st century: from early detection to new therapies. Radiología (English Edition), 59(5), 368-379.

Chansky, K., Benedetti, J., \& Macdonald, J. S. (2005). Differences in toxicity between men and women treated with 5-fluorouracil therapy for colorectal carcinoma. Cancer: Interdisciplinary International Journal of the American Cancer Society, 103(6), 1165-1171.

Chaveli-López, B., \& Bagán-Sebastián, J. V. (2016). Treatment of oral mucositis due to chemotherapy. Journal of Clinical and Experimental Dentistry, 8(2), e201.

Cleverson, P., Laurindo Moacir, S., Eduardo Ciliao, M., Roberta Targa Stramandinoli, Z., \& Juliana Lucena, S. (2014). Clinical assessment of oral mucositis and candidiasis compare to chemotherapic nadir in transplanted patients. Brazilian Oral Research, 28(1), 1-7.

Curra, M., Soares Junior, L. A. V., Martins, M. D., \& Santos, P. S. D. S. (2018). Chemotherapy protocols and incidence of oral mucositis. An integrative review. Einstein (São Paulo), 16(1).

Daugèlaitė, G., Užkuraitytė, K., Jagelavičienė, E., \& Filipauskas, A. (2019). Prevention and treatment of chemotherapy and radiotherapy induced oral mucositis. Medicina, 55(2), 25.

Dodd, M. J., Miaskowski, C., Shiba, G. H., Dibble, S. L., Greenspan, D., Macphail, L., \& Larson, P. (1999). Risk factors for chemotherapy-induced oral mucositis: dental appliances, oral hygiene, previous oral lesions, and history of smoking. Cancer Investigation, 17(4), $278-284$.

Elad, S., Cheng, K. K. F., Lalla, R. V., Yarom, N., Hong, C., Logan, R. M., \& Zur, E. (2020). MASCC/ISOO clinical practice guidelines for the management of mucositis secondary to cancer therapy. Cancer, 126(19), 4423-4431.

Holmes, T. S. V., dos Santos, M. G. C., de Medeiros Nóbrega, D. R., Pereira, J. V., de Castro Gomes, D. Q., \& Pereira, M. D. S. V. (2014). Fatores relacionados ao surgimento e gradação da mucosite oral radioinduzida. Revista Cubana de Estomatología, 51(1), 45-51.

Hong, B. Y., Sobue, T., Choquette, L., Dupuy, A. K., Thompson, A., Burleson, J. A., \& Diaz, P. I. (2019). Chemotherapy-induced oral mucositis is associated with detrimental bacterial dysbiosis. Microbiome, 7(1), 1-18.

Hong, C. H., Gueiros, L. A., Fulton, J. S., Cheng, K. K. F., Kandwal, A., Galiti, D., \& Elad, S. (2019). Systematic review of basic oral care for the management of oral mucositis in cancer patients and clinical practice guidelines. Supportive Care in Cancer, 27(10), 3949-3967.

Jesus, L. G. D., Cicchelli, M., Martins, G. B., Pereira, M. C. C., Lima, H. S., \& Medrado, A. R. A. P. (2016). Oral effects of anticancer drugs: a literature review. Revista da Faculdade de Odontologia - UPF, 21(1), 130-135.

Kusiak, A., AlicjaJereczek-Fossa, B., Cichońska, D., \& Alterio, D. (2020). Oncological-therapy related oral mucositis as an interdisciplinary problem-Literature review. International Journal of Environmental Research and Public Health, 17(7), 2464.

Lalla, R. V., Sonis, S. T., Peterson, D. E (2008). Management of Oral Mucositis in Patients with Cancer. Dent Clin North Am, 52(1), 61-77.

McCarthy, G. M., Awde, J. D., Ghandi, H., Vincent, M., \& Kocha, W. I. (1998). Risk factors associated with mucositis in cancer patients receiving 5-fluorouracil. Oral Oncology, 34(6), 484-490.

Morais, J. R., Vanderley, T. R., Soares, R. D. M., Castro, A. E. D., Araújo, S. N. M., \& Luz, M. H. B. A. (2015). Characterization of the patient with chemotherapy-induced oral mucositis/Caracterização do paciente com mucosite oral induzida por quimioterapia/Caracterización de la paciente con mucositis oral inducida por quimioterapia. Revista de Enfermagem da UFPI, 4(1), 26-32.

National Cancer Institute. (2017). Common Terminology Criteria for Adverse Events (CTCAE). United State Department of Health and Human Services, v.5.0.

Park, S. H., \& Lee, H. S. (2019). Meta-analysis of oral cryotherapy in preventing oral mucositis associated with cancer therapy. International Journal of Nursing Practice, 25(5), e12759.

Pereira, I. F., Firmino, R. T., Meira, H. C., Vasconcelos, B. C. D. E., Noronha, V. R. A. D. S., \& Santos, V. R. (2019). Radiation-induced oral mucositis in Brazilian patients: Prevalence and associated factors. In Vivo, 33(2), 605-609. 
Pulito, C., Cristaudo, A., La Porta, C., Zapperi, S., Blandino, G., Morrone, A., \& Strano, S. (2020). Oral mucositis: the hidden side of cancer therapy. Journal of Experimental \& Clinical Cancer Research, 39(1), 1-15.

Raber-durlacher, J. E., Elad, S., Barasch, A. (2010). Oral mucositis. Oral Oncology, 46 (6), 452-456.

Rother, Edna Terezinha. (2007). Revisão sistemática X revisão narrativa. Acta Paulista de Enfermagem, 20(2), v-vi. https://doi.org/10.1590/S010321002007000200001

Saedi, H. S., Gerami, H., Soltanipour, S., Habibi, A. F., Mirhosseyni, M., Montazeri, S., \& Nemati, S. (2019). Frequency of chemoradiotherapy-induced mucositis and related risk factors in patients with the head-and-neck cancers: A survey in the North of Iran. Dental Research Journal, 16(5), 354.

Scully, C., Sonis, S., Diz, P. D. (2006). Oral mucositis. Oral Diseases, 12(3), 229-241.

Seiler, S., Kosse, J., Loibl, S., \& Jackisch, C. (2014). Adverse event management of oral mucositis in patients with breast cancer. Breast Care, 9(4), $232-237$.

Shankar, A., Roy, S., Bhandari, M., Rath, G. K., Biswas, A. S., Kanodia, R., \& Sachan, R. (2017). Current trends in management of oral mucositis in cancer treatment. Asian Pacific Journal of Cancer Prevention: APJCP, 18(8), 2019.

Sloan, J. A., Goldberg, R. M., Sargent, D. J., Vargas-Chanes, D., Nair, S., Cha, S. S., \& Loprinzi, C. L. (2002). Women experience greater toxicity with fluorouracil-based chemotherapy for colorectal cancer. Journal of Clinical Oncology, 20(6), 1491-1498.

Sonis, S. T. (2009). Mucositis: the impact, biology and therapeutic opportunities of oral mucositis. Oral Oncology, 45(12), 1015-1020.

Sonis, S. T., Lieberman, A., \& Sonis, A. L. (1978). Oral complications in patients receiving treatment for malignancies other than of the head and neck. The Journal of the American Dental Association, 97(3), 468-472.

Sonis, S., \& Clark, J. (1991). Prevention and management of oral mucositis induced by antineoplastic therapy. Oncology (Williston Park, NY), 5(12), 11-8.

Suresh, A. V. S., Varma, P. P., Sinha, S., Deepika, S., Raman, R., Srinivasan, M., \& Anand, B. B. (2010). Risk-scoring system for predicting mucositis in patients of head and neck cancer receiving concurrent chemoradiotherapy. Journal of Cancer Research and Therapeutics, 6(4), 448.

Thorne S. Rediscovering the "Narrative" review. Nursing Inquiry. 25(3):e12257. 10.1111/nin.12257. PMID: 30003651.

Watanabe, T., Kuranami, M., Inoue, K., Masuda, N., Aogi, K., Ohno, S., \& Ohashi, Y. (2017). Comparison of an AC-taxane versus AC-free regimen and paclitaxel versus docetaxel in patients with lymph node-positive breast cancer: Final results of the National Surgical Adjuvant Study of Breast Cancer 02 trial, a randomized comparative phase 3 study. Cancer, 123(5), 759-768.

Wilairat, P., Kengkla, K., Kaewpanan, T., Kaewthong, J., Ruankon, S., Subthaweesin, C., \& Saokaew, S. (2020). Comparative efficacy and safety of interventions for preventing chemotherapy-induced oral mucositis in adult cancer patients: a systematic review and network meta-analysis. European Journal of Hospital Pharmacy, 27(2), 103-110.

Yu, Y. Y., Deng, J. L., Jin, X. R., Zhang, Z. Z., Zhang, X. H., \& Zhou, X. (2020). Effects of 9 oral care solutions on the prevention of oral mucositis: a network meta-analysis of randomized controlled trials. Medicine, $99(16)$.

Zhang, X., Sun, D., Qin, N., Liu, M., Zhang, J., \& Li, X. (2020). Comparative prevention potential of 10 mouthwashes on intolerable oral mucositis in cancer patients: A Bayesian network analysis. Oral Oncology, 107, 104751.

Zhang, X. H., Hao, S., Gao, B., Tian, W. G., Jiang, Y., Zhang, S., \& Luo, D. L. (2016). A network meta-analysis for toxicity of eight chemotherapy regimens in the treatment of metastatic/advanced breast cancer. Oncotarget, 7(51), 84533-84543. 